\title{
Automatic Movable Smart Road Dividers - IOT Based Solution to Traffic Congestion Problems
}

\author{
${ }^{1}$ Keerthan A J, ${ }^{2}$ Likhith H D, ${ }^{3}$ Chetan H S, ${ }^{4}$ Kiran K P, ${ }^{5}$ Dr. Sumaiya MN \\ ${ }^{*} 1,2,3,4$ Students, Dayananda Sagar Academy of Technology \& Management, Bangalore, India. \\ *5 Associate Professor, ECE Department, Dayananda Sagar Academy of Technology \& Management, Bangalore, India.
}

\begin{abstract}
The street dividers are commonly utilized for separating the street for continuous and approaching traffic. This is useful in keeping the progression of traffic. For this situation, there is equal number of lanes for both approaching and continuous traffic. Be that as it may, in certain zones, such as in industrial or shopping zones the traffic for the most part streams a one way in the first part of the morning or night. The other roadside is unfilled or unused. It leads to time loss for public and congested driving conditions. We mean to assemble a shrewd street divider as far as a mechanized street divider which moves or move the path coordinating the surge in rush hour gridlock. Such kind of component of traffic framework spares time as well as fuel. It can include 1 more path based the traffic in the specific bearing. With the more intelligent application planned underneath, manual reliance and manual traffic relation is decreased. Like this a proposition of savvy traffic is worked in less, medium and more density in the rush hour gridlock will be appeared in IOT server in utilizing grap diagram. IoT refers to Internet of Things where real digitalization comes into the image. The sensors and Arduino boards are utilized. The sensors are placed on the dividers which sense the progression of traffic and it is refreshed to the web through Wi-Fi module associated. The traffic density will be appeared in IoT server in utilizing graph chart. It gives a superior answer to traffic issue.
\end{abstract}

Keywords:- Mechanized street divider, traffic framework, IoT server, Sensors, RFID.

\section{INTRODUCTION}

Nations around the globe are confronting issue of traffic problem because of increment in the number of vehicles. In spite of the fact that the quantity of vehicles utilizing the streets has been expanded, the static street foundation is nearly the equivalent and it can't adapt to the progressions like a blockage. The problem with static street divider is that the number of lanes on either side of the streets are fixed. whereas the assets are restricted and there is a critical increment in the number of vehicles on the street. This calls for better utilization of existing assets like a number of paths accessible. So as to conquer this issue, we structure a portable street divider which moves to rely upon the progression of traffic. The sensors can be worked by the microcontroller to recover the information from them and update it to the web through Wi-Fi module associated with it. The IoT gathers the continuous information of vehicular which traffic that discovers the present traffic activity and traffic stream condition. The IoT will be associated with every single piece of traffic, for example, streets, dividers with the assistance of infrared sensors. As a rule, we see that there will be immense traffic on one side of the divider of a street and on the opposite side, there will be no traffic. In this sort of circumstance, it is conceivable to control the divider position consequently which decreases the traffic issues. The movable street divider helps in the arrangement of the street limit, in order to achieve ideal profit by the roadway utilization on the current street. By utilizing the development of divider, we can give traffic clearance for the emergency vehicle when it required.

\section{MOTIVATION}

Our idea is to calculate an instrument of robotized compact road divider that can move ways, so we can have a progressive number of ways toward the rush. The total impact of the time and fuel that can be saved by adding even one extra way to control traffic congestion. With the sagacious application proposed underneath, we will moreover discard the dependence on manual intercession and manual traffic coordination so we can have progressively wise traffic wherever all through the city.

\section{LITERATURE REVIEW}

In [1], a versatile Traffic Management System (TMS) joined with a fuzzy rationale-based plan so as to take proper activities to accelerate the advancement of crisis vehicles while maintaining a strategic distance from the production of bottlenecks around their courses. This is accomplished through the all-around planned adjustment activities and crisis reaction plans picked dependent on the crisis level promoted by the crisis vehicle and the yield of the fuzzy framework.

In [2], proposed a innovation in robotizing the traffic signals by utilizing picture handling, Infrared sensor, and in some spot's prioritization in rush hour gridlock motioning towards crisis vehicles dependent on the fuzzy rationale. The test with every one of these frameworks is that it is pricey.

In [3], they proposed image capturing process instead of sensing vehicles by sensors. In this system traffic light can be control by using image processing method. By using analyse data traffic signal can be controlled. With the assistance of specific calculation, morphology and picture are expected to stay away from traffic blockage. 
In [4], RFID is a remote connection to extraordinarily recognize objects or people. RFID empowers distinguishing proof from a separation without requiring a view. This disposes of the utilization of additional equipment. This likewise incorporates the acknowledgment of vehicles by the sound of their alarm. This framework stays away from the meddle in the rush hour gridlock stream the board. By utilizing the RFID tag, a different way will be make for emergency vehicles.

In [5], proposed an intelligent traffic organization framework, in view of the Internet of Things, which is included by minimal effort, high versatility, high similarity, easy to upgrade, to replace conventional traffic the executive's framework and the proposed framework can improve street traffic enormously.

In [6], the traffic parameters, for example, traffic volume, path speed, path inhabitancy, and vehicle progress are gauges and utilized. The day of the week and the hour of day are additionally utilized by the calculation. Information investigation is done on a for every path premise and results are created for every path and for the general street fragment. Improving the proficiency of Traffic Management Systems (TMS) is as yet a functioning and testing research territory because of the criticality of transportation foundation being observed by such system. In [7-9], various smart and intelligent vehicle systems have been discussed.

\section{MATERIALS AND METHODOLOGY}

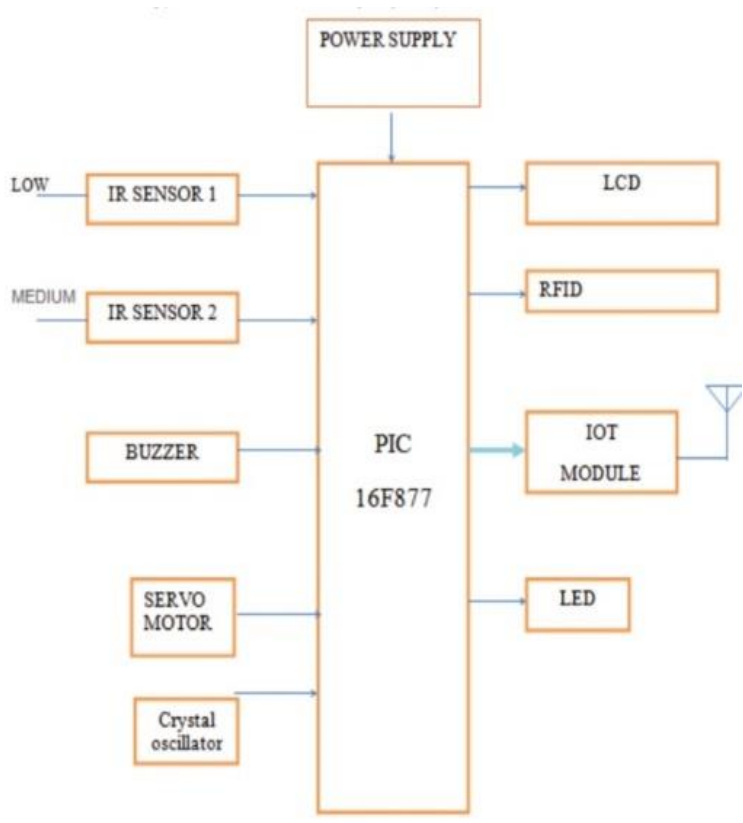

Fig 1:- The block diagram of the proposed system.

The executed framework comprises of microcontroller as a principle preparing unit and all the sensors and gadgets are associated with the microcontroller. The sensors can be worked by the microcontroller to recover the information from them and update it to the web through Wi-Fi module associated with it.

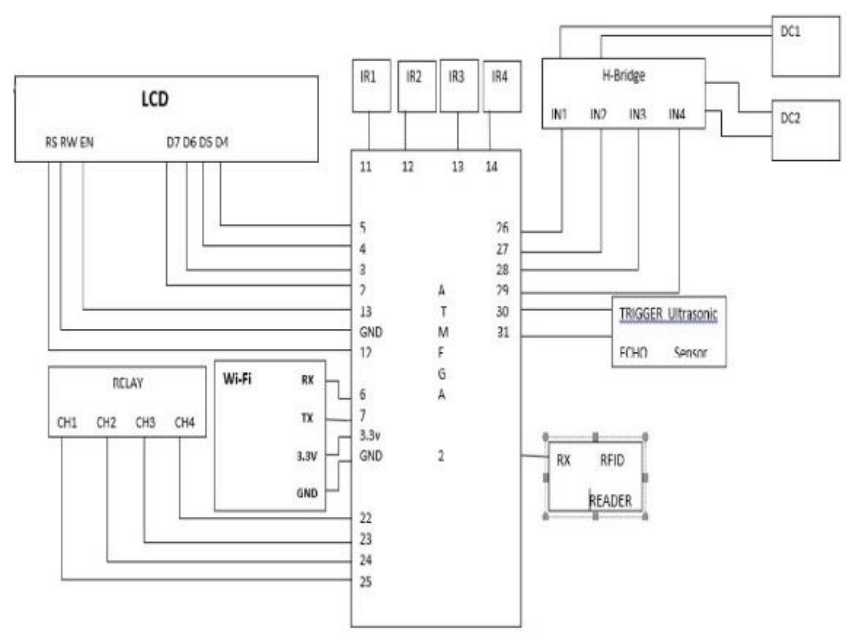

Fig 2:- Circuit diagram

\section{ARDUINO:}

Arduino is an open source equipment and programming. It has 14 advanced input/output pins, 6 simple data sources, a $16 \mathrm{MHz}$ artistic resonator, a USB association, a force jack, an ICSP header, and a reset button. It contains everything expected to help the microcontroller. The ATMEGA328 on the Arduino UNO comes preprogrammed with a boot loader that permits us to transfer new code to it without utilizing an external hardware programmer.

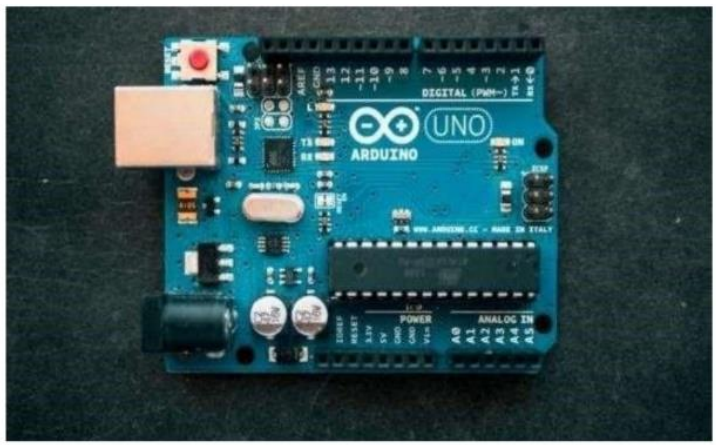

Fig 3:- Arduino

\section{IR SENSORS:}

An infrared sensor is an electronic gadget that produces so as to detect a few parts of the environmental factors. An IR sensor can measure the hear of an object as well as identifies the movement.

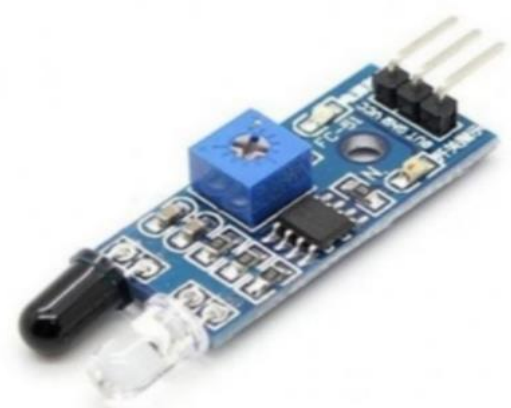

Fig 4:- IR Sensor 


\section{ESP8266 Wi-Fi Module:}

ESP8266 is Wi-Fi enable system on chip(SOC) module created by Espressif framework. It is for the most part utilized for the advancement of IoT implanted applications. It eases independent remote handset that can be utilized for end-point IoT advancements. It has $2.4 \mathrm{GHz}$ Wi-Fi, 16 GPIO, 10 piece ADC, Inter-Integrated Circuit serial communication protocol.

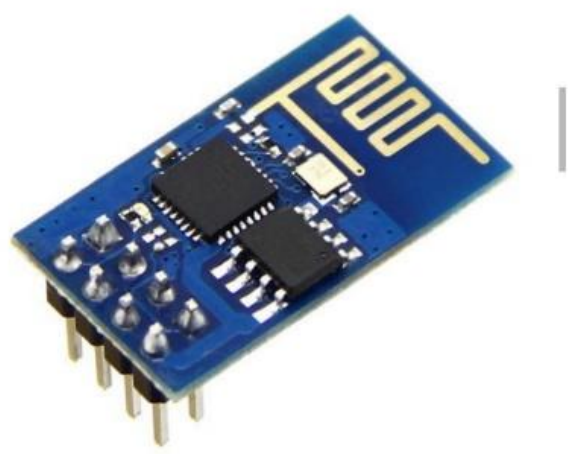

Fig 5:- ESP8266 Wi-Fi Module

\section{MOTOR:}

A DC motor is an electric motor that runs on direction current. It chips away at the way that a current conveying conductor set in an attractive field encounters a power which makes it turn as for its original position.

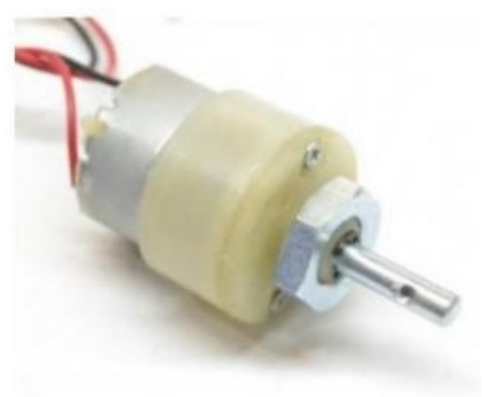

Fig 6:- DC Motor

\section{L293D:An H-Bridge:}

A H-Bridge is only an electronic circuit. Utilizing such circuit, we can make current to supply in two ways. The L293D is an H-Bridge with two potential outputs. Two things can be associate with it and the bearing of the current stream can be controlled in both.

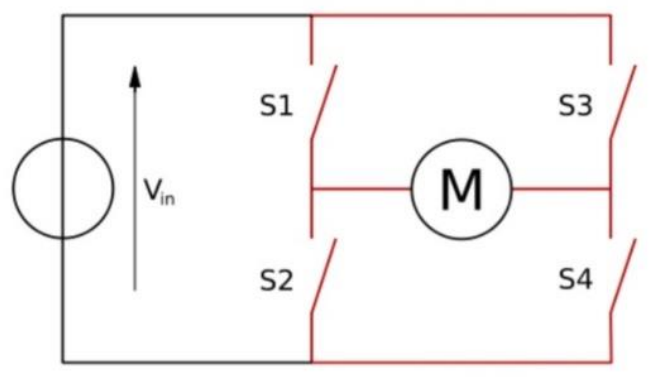

Fig 7:- L293D H-Bridge

\section{LIQUID CRYSTAL DISPALY:}

An LCD is a flat board show or another electronically adjusted optical gadget that utilizes the light-balancing properties of fluid precious stones consolidates with polarizers. Fluid precious stones don't emanate light directly, rather utilizing a backdrop illumination or reflector to deliver pictures in shading or monochrome.

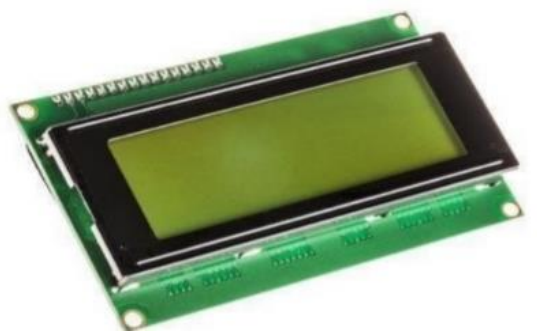

Fig 8:- LIQUID CRYSTAL DISPALY

$>$ RFID:

RFID is a remote connection to interestingly distinguish objects or people. It enables recognizable proof from a distance without requiring a view. The RFID framework contains the RFID tag/card, RFID reader, backend database and a control unit. RFID frameworks have two general classes active and passive. The RFID reader communicates with the RFID tag through label cross-examination.

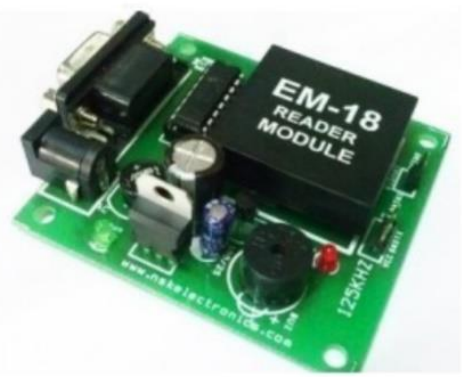

Fig 9:- RFID

The fundamental point in the planed structure is to diminish the reaction time to emergency or government vehicles, for instance, emergency vehicle, government, police, and firefighters vehicles and so forth., by giving RFID tag to these vehicles. The proposed technique is clarified utilizing the stream diagram as appeared in figure underneath. The suggested structure shows that the vehicle out and about are taken in tally and relying upon the power of traffic the divider moves. Two sorts of sensors are used here normal and high types The data acquired from sensors are refreshed in the site through the $\mathrm{Wi}-\mathrm{Fi}$ module and showed on LCD. At the point when an emergency vehicle with an RFID label goes through each observing station along with the road, the RFID reader at those focuses will consequently read the label information identified with the emergency vehicle and gives the path by moving the divider. At the point when system interfaces with the web, all the information about traffic conditions on each street area are immediately saved in the database which is storage point and can be used in any way, shape ,or form and application. 
ISSN No:-2456-2165 Fig.3.

The flowchart of the proposed system is shown in

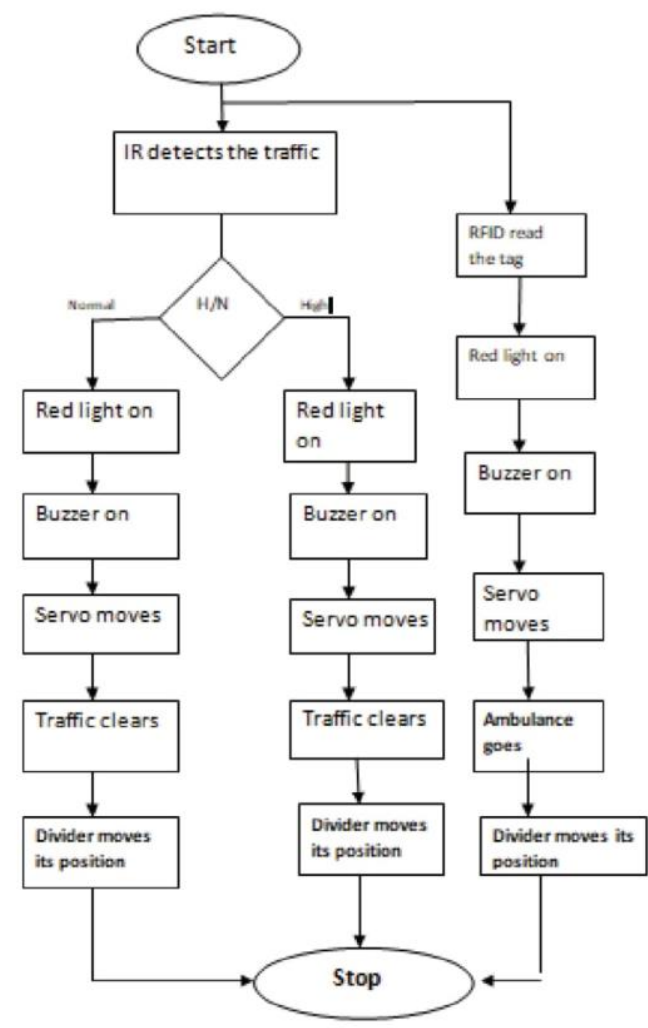

Fig 10:- The flowchart.

\section{SIMULATION RESULTS}

The simulation results at various stages have been shown in Fig. 4 to Fig.9

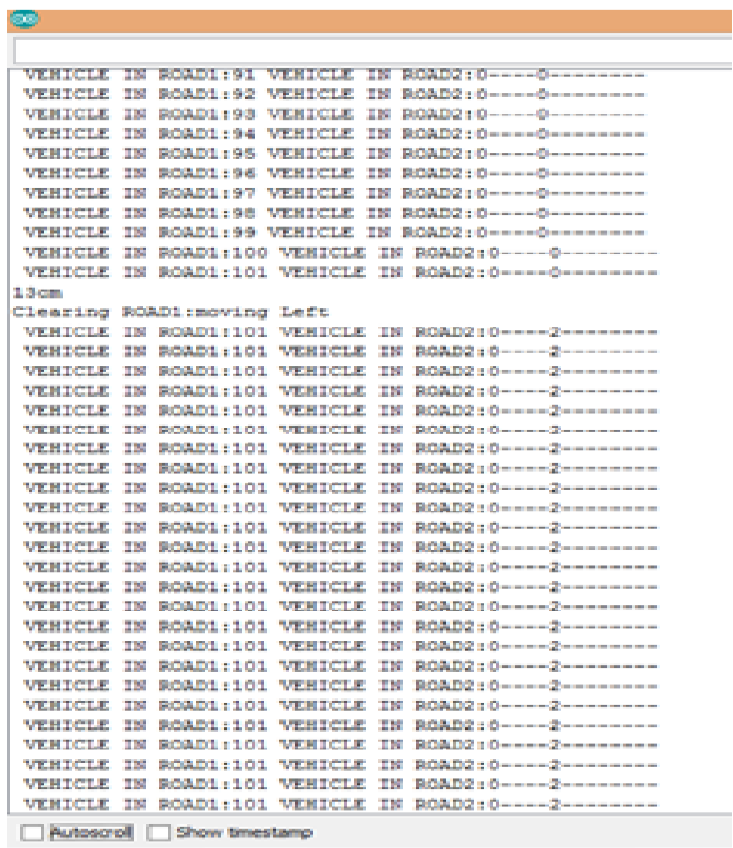

Fig 11:- Screenshot of divider moving Left

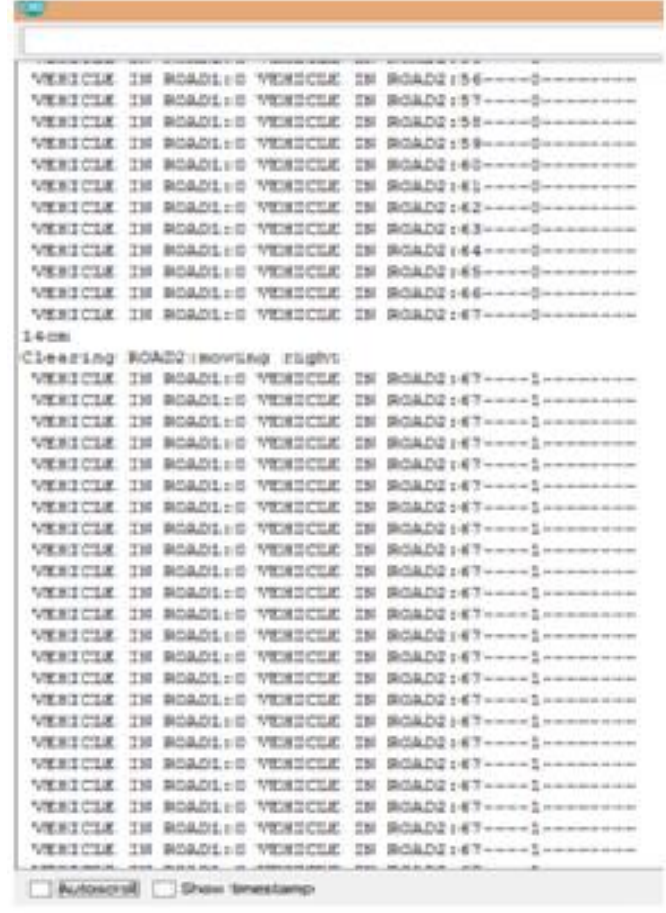

Fig 12:- Screenshot of divider moving Right

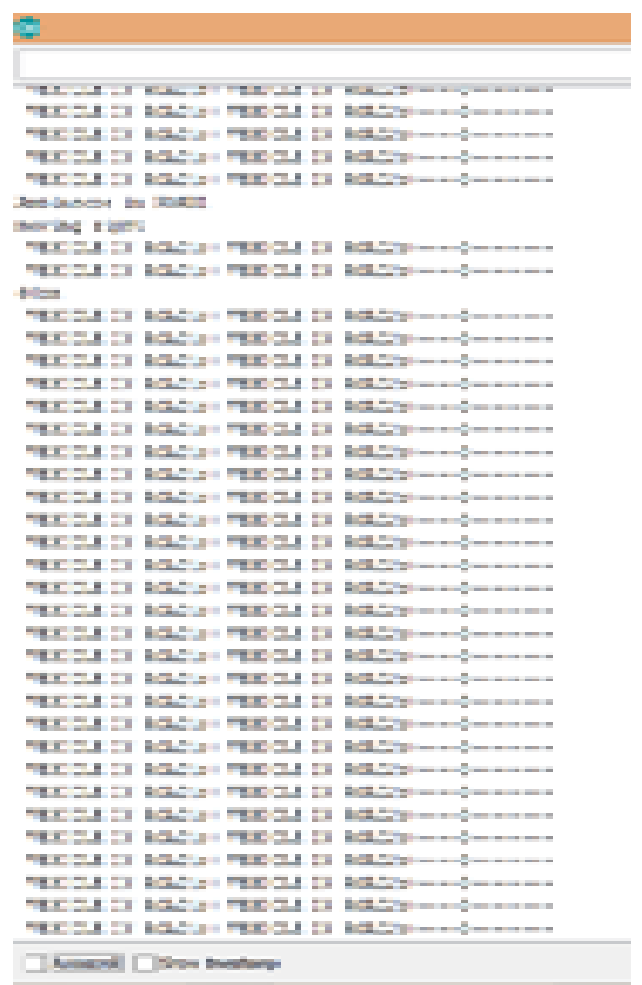

Fig 13:- Screenshot of divider moving Right for making way to Ambulance in Road1 


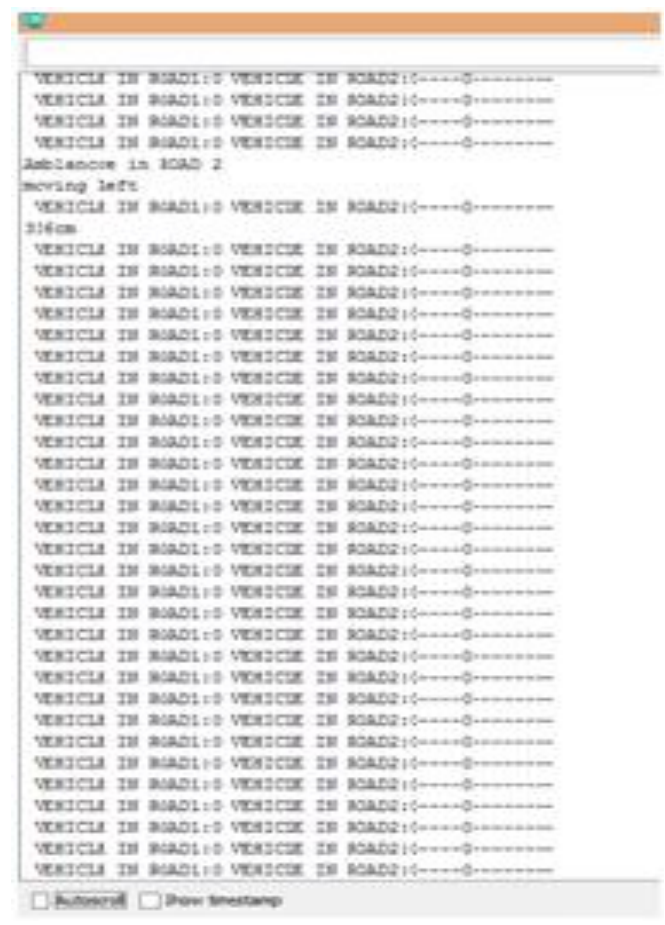

Fig 14:- Screenshot of divider moving Left for making way to Ambulance in Road2

\section{TCP CLIENT}

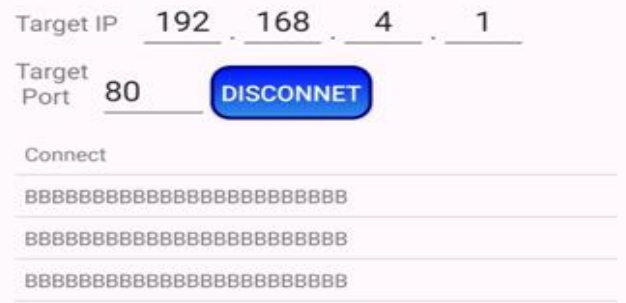

repeat BBBBBBBBBBBBBB BBBBBBBBBB

\section{SEND}

\section{Kᄌ Tap full-screen display}

Fig 15:- Screenshot of giving the input for making way to Ambulance in Road1

\section{TCP CLIENT}

\section{$\vdots$}

repeat AAAAAAAAAAAA AAAAA

\section{KᄌT Tap full-screen display}

Fig 16:- Screenshot of giving the input for making way to Ambulance in Road2

At First before moving the divider the ultrasonic makes sure there no vehicles near the road divider, IF any vehicles present, the buzzer starts alarming till the vehicle is moved. IR sensor will check the number of vehicles in microseconds, as soon as the count above 50.The divider starts moving according to density of the vehicles in roads.

Examples If the density of vehicles is more in ROAD1, the divider moves to left (opposite site of Road1) making the RGB LED's to GREEN, making the traffic Signal in ROAD1 to GREEN to make the vehicles should to pass out as soon as possible. RGB LED's present in ROAD2 will be RED \& traffic signal in Road2 will be RED.

USING the Wi-Fi Module the User (Ambulance Driver or Traffic Police) gives the input " $\mathrm{A}$ " or " $\mathrm{B}$ " in TDP/UDP Application in mobile making the road move accordingly .

\section{CONCLUSION}

Here undertaking, the road is related with a cloud which steady seeing of the traffic is done and the density of traffic is moved to the cloud. Traffic density which is open in the cloud can be used for a various purposes like traffic writes about various applications for example, here maps. In the wake of transferring traffic reports on a cloud by considering traffic force in three factors like LESS, MEDIUM, and the MORE road divider is moved by a small distance. In the event that force is LOW, at that point 
divider remains in its position. If the intensity is MEDIUM then divider moves by a little separation. In the event that intensity is $\mathrm{HIGH}$, at that point divider moves by an enormous separation. The project additionally gives an answer for traffic clearance to the emergency and government vehicle. Utilizing RFID a cloud is made to distinguish the appearance of an emergency vehicle and afterward to make a path uniquely for emergency vehicles by moving street divider in a like manner. Henceforth it is presumed that it is conceivable to dodge blockage in a given course by moving the street divider to extend or limit the street and clear the traffic. Additionally, it is conceivable to give a free route to the emergency vehicle independent of the traffic out and about.

\section{REFERENCES}

[1]. Soufiene Djahel "Reducing Emergency Services Response Time in Smart Cities: An Advanced Adaptive and Fuzzy Approach", IEEE 2015.

[2]. R. Weil, J. Wootton and A. Garcia Ortiz, " Traffic Incident Detection Sensor and Algorithms", Journal of Mathematical and Computer Modelling, Vol.27 (9), 1998, pp.257-291.

[3]. Roopa Ravish, Varun R. Gupta ,K J Nagesh "Software Implementation of an Automatic Movable Road Barrier" 2019 International Carnahan Conference on Security Technology(ICCST).

[4]. Satya Srikanth, H B Vibha, B M Sriraksha, A Yashashwini "Implementation of Smart Movable Road Divider and Ambulance Clearance using IoT" $20194^{\text {th }}$ International Conference on Recent Trends on Electronics ,Information,Communicatio n, and Technology(RTEICT)

[5]. B Durga Sri, K Nirosha, Sheik Gouse "Design and Implementation of small movable road divider using IoT " 2017 International Conference on Intelligent Sustainable Systems(ICISS).

[6]. Ch V A Satwik, L Pavan Kumar,K Vineeth, Kavitha N P Pillai "Intelligent Road Management System for Daily Transit" 2018 International Conference on Communication and Signal Processing(ICCSP).

[7]. C.N.Shreyas, V.Srivasta, Vaibhav Pitale, M.Ananda "IoT Based Smart Signal" $20182^{\text {nd }}$ International Conference on I-SMAC.

[8]. P.Kalaivani, R.Pretheep Kumar, J.Thrshan, R.Shakthi Kolapan "Smart collision avoidance and driver alert recognition(SCADAR) system $2017 \quad$ Third International Conference on Sensing, Signal Processing and Security . 